\title{
O Processo de Cuidados: análise da conceção dos estudantes de Enfermagem
}

\author{
The Care Process: analysis of Nursing students' conceptions \\ El proceso de atención: análisis de la concepción de los estudiantes de Enfermería
}

Ana Cristina Spínola*; José Amendoeira**

\section{Resumo}

Enquadramento: A Formação em Enfermagem exige processos pedagógicos inovadores, com estratégias promotoras da aprendizagem dos estudantes nos contextos de formação, estas deverão ser facilitadoras do seu desenvolvimento no processo de cuidados.

Objetivos: Analisar a conceção do processo de cuidados em ensino clínico, recorrendo a uma estratégia informática especifica.

Principais tópicos em análise: Analisámos 30 reflexões críticas de estudantes do $4^{0}$ ano de Enfermagem, utilizando o Microsoft Word 2010; selecionámos 34 palavras com base em três critérios: sentido semântico; frequência; e contextualização teórica.

Da estrutura cognitiva dos estudantes emergiram três temas: conceção dos cuidados; intervenção de Enfermagem; e aprendizagem do processo de cuidados. Identificámos palavras plenas de sentido: cuidados; pessoa; ajudar; momentos; avaliação; intervenção; competências; aprendizagem; e reflexão.

Representámos as palavras identificadas em mapa conceptual do processo de cuidados (CmapTools).

Conclusão: O processo pedagógico promove um continuum na aquisição de competências pelos estudantes. Identificámos a conceção do processo de cuidados em ensino teórico relacionada com o pensamento crítico em ensino clínico. Implicação do estudo: utilização do mapa conceptual como estratégia pedagógica promotora da autorreflexão nos estudantes.

Palavras-chave: estudantes de Enfermagem; cuidados de Enfermagem; aprendizagem.

\begin{abstract}
Background: Nursing Education requires innovative pedagogical processes, with strategies that promote the students' learning in training contexts. These strategies should facilitate the students' development in the care process.

Aims: To analyse the conception of the care process in clinical teaching, using a specific computer strategy.

Main topics for analysis: Thirty critical reflections of $4^{\text {th }}$-year nursing students were analysed using Microsoft Word 2010. Thirty-four words were selected based on three criteria: semantic meaning, frequency, and theoretical background.

Three themes emerged from the students' cognitive structure: conception of care, nursing intervention, and care process learning. Full-meaning words were identified: care, person, help, moments, assessment, intervention, skills, learning, and reflection. The identified words were represented in a concept map of the care process (CmapTools).

Conclusion: The pedagogical process promotes a continuum in the students' acquisition of skills. We identified the conception of the care process in theoretical training related to critical thinking in clinical teaching. Study implication: use of the concept map as a pedagogical strategy to promote self-reflection in students.
\end{abstract}

Keywords: students, nursing; nursing care; learning.

* Professora Adjunta do Instituto Politécnico de Santarém - Escola Superior de Saúde, 2005 - 075 Santarém, Portugal. Doutoranda em Ciências de Enfermagem, ICBAS-Universidade do Porto. Mestre em Ciências de Educação (2004). Licenciada em Enfermagem na Comunidade [aspinola748@gmail.com]. Morada para correspondência: Rua da Escola Nova n ${ }^{\circ} 35,2125-135$, Marinhais, Portugal.

* Professor Coordenador do Instituto Politécnico de Santarém - Escola Superior de Saúde, 2005 -075 Santarém, Portugal. Investigador da UiIPS, PhD, [jjpam2@ gmail.com]

\section{Resumen}

Contexto: La formación en enfermería requiere procesos pedagógicos innovadores, con estrategias que promuevan el aprendizaje del estudiante en los contextos de formación, que deben facilitar su desarrollo en el proceso de atención.

Objetivos: Analizar el diseño del proceso de atención en la enseñanza clínica, utilizando una estrategia informática específica. Principales temas de análisis: Se analizaron 30 reflexiones críticas de estudiantes del cuarto año de enfermería, utilizando para ello Microsoft Word 2010, y se seleccionaron 34 palabras de acuerdo con tres criterios: sentido semántico, frecuencia y contexto teórico. La estructura cognitiva de los estudiantes revela tres temas: diseño de la atención, intervención de enfermería y aprendizaje del proceso de atención.

Se identificaron palabras llenas de significado: cuidado, persona, ayuda, momentos, evaluación, intervención, competencias, aprendizaje, reflexión. Representamos las palabras identificadas en un mapa conceptual del proceso de atención (Cmaptools).

Conclusión: El proceso educativo promueve un continuum en la adquisición de competencias por los estudiantes. Identificamos: diseño del proceso de atención en la enseñanza teórica relacionada con el pensamiento crítico en la enseñanza clínica. Implicación del estudio: el uso del mapa conceptual como estrategia pedagógica promueve la autorreflexión en los estudiantes.

Palabras clave: estudiantes de Enfermería; atención de enfermería; aprendizaje.

Recebido para publicação em: 15.01 .14

Aceite para publicação em: 25.03 .14 


\section{Introdução}

Atualmente na formação em Enfermagem, os processos pedagógicos refletidos no planeamento das estratégias, no decurso da aprendizagem dos estudantes em vários contextos de formação, assumem-se como determinantes na aquisição do conhecimento e desenvolvimento de competências a partir da prática de cuidados (Ordem dos Enfermeiros, 2007; Decreto-Lei no 115/13).

A complexidade crescente dos contextos, bem como os próprios cuidados desenvolvidos, exigem que a formação em Enfermagem seja um processo de integração/transposição de saberes pelos estudantes em cada contexto. Esta transposição é tão mais consistente quanto melhor for realizada a alternância entre a escola e as organizações de saúde (Amendoeira, 2009; Rua, 2011).

Interessou-nos o ensino clínico refletido na formação em Enfermagem e desenvolvido nas organizações de saúde, com o seu potencial inovador e como estratégia de aquisição de aprendizagens significativas pelos estudantes (Costa, 2008). Visámos a identificação dos conhecimentos adquiridos pelos estudantes através das suas reflexões sobre as práticas desenvolvidas.

O relacionamento entre os atores envolvidos no contexto de cuidados é determinante, considerando os estudantes, professores, enfermeiros e a pessoa sujeito dos cuidados. Por outro lado, a exigência que as atividades educativas assumem para o estudante decorrem de a ela estarem ligados procedimentos com atitudes, normas e valores que fazem parte de uma organização de saúde (Carvalho, 2004).

Todavia, sabemos que a Enfermagem é uma profissão centrada em interações onde cada pessoa, face ao seu projeto de vida, se torna singular num momento único de cuidado (Serrano, Costa, \& Costa, 2011). Para o estudante estes momentos são únicos e determinantes na reflexão e na aquisição de saberes na prática clínica com a tomada de consciência do cuidado produzido, procurando a seleção das melhores metodologias para responder às necessidades em função dos sujeitos de cuidados de Enfermagem (Abreu, 2007). Neste sentido, os saberes profissionais do estudante vão sendo construídos através da interação com os profissionais de saúde e pela análise que faz das práticas quotidianas dos enfermeiros, relacionando-as com os saberes teóricos numa aliança na procura do saber (Costa, 2008).
Trata-se portanto do processo de aprendizagem em ensino clínico, desenvolvido a par do crescimento e autonomia pessoal, alicerçado na experiência de vida do estudante, associada ao seu itinerário sociocultural e das representações que construíram de si próprios e do seu meio humano e natural (Josso, 2002). Parece-nos portanto fundamental a compreensão do processo de aprendizagem que o estudante desenvolve para que seja possível a aquisição de competências de carácter científico, técnico e humano, de forma a ser capaz de planear, concretizar, gerir e avaliar atividades de Enfermagem (Carvalho, 2004). Por outro lado, torna-se necessário caracterizar o processo de cuidados que o estudante desenvolve em ensino clínico, tal como refere Amendoeira (2000, 2004). Integramos a diversidade das atividades de Enfermagem desenvolvidas pelos estudantes, tendo por base a interação entre estes e as pessoas alvo dos cuidados; consideramos ainda os conhecimentos específicos que lhes permitem diagnosticar e planear o trabalho, que eles próprios executam e controlam. Nesta perspetiva, entre as diferentes atividades que 0 estudante desenvolve no ensino clínico e a vivência das suas diversas experiências, ocorrem inevitavelmente aprendizagens significativas no percurso de formação. Neste sentido, mais do que aprendizagem, o ensino clínico implica necessariamente um trabalho de conceptualização da prática (Costa, 2008), tornando-se necessário compreendermos que aquisições teóricas são desenvolvidas e como surgem enquadradas as conceções dos estudantes quando cuidam de pessoas.

Nem sempre a forma como é produzido o processo de cuidados pelos estudantes em ensino clínico deixa claras as conceções mobilizadas a partir do ensino teórico. Sendo este o foco deste estudo, pretendemos analisar a conceção do processo de cuidados desenvolvido pelo estudante de Enfermagem em ensino clínico, a partir de uma estratégia pedagógica inovadora com recurso informático.

\section{Dissertação}

Na Formação em Enfermagem, o processo de aprendizagem pelo estudante em contexto de ensino clínico desenvolve-se para e com o sujeito de cuidados, sustentado em conhecimento científico, habilidades, pensamento crítico, e comportamentos e 
atitudes que vão no sentido de promover o bem-estar. Ao admitirmos um paradigma centrado no estudante enquanto sujeito aprendente, perspetivamos a sua aprendizagem com o recurso à utilização das próprias experiências, visando a reflexão e a identificação das dimensões e dos momentos importantes para o desenvolvimento do processo de auto e héteroformação, em interação com os diferentes atores, em contextos de ensino clínico (Josso, 2002).

Reconhecemos que são os contextos de trabalho que atribuem uma significação às práticas de saúde pelo estudante, no sentido em que estes estão dependentes de uma dinâmica cognitiva e avaliativa definida em contexto, em contacto com um conjunto de fatores materiais, relacionais e simbólicos inerentes ao funcionamento duma organização de saúde (Abreu, 2007).

Por outro lado, nos contextos da prática clínica, são diversos os atores que se constituem como promotores do desenvolvimento dos estudantes, nomeadamente os professores, os enfermeiros supervisores, os profissionais da equipa de saúde e os próprios sujeitos de cuidados (Amendoeira, 2009; Rua, 2011). Este desenvolvimento é particularmente importante quando pensamos numa aliança pedagógica que se constitui como facilitadora da aprendizagem do estudante porque, por um lado, conhecem os contextos da prática, e por outro, são detentores dos conhecimentos/saberes essenciais à facilitação desse mesmo desenvolvimento. Desta forma, torna-se fundamental que todos os atores em ensino clínico se envolvam na formação dos estudantes na aquisição de conceções de cuidados consentâneos com o paradigma de formação atual, tornando os estudantes o centro e o motor de todo o ensino em Enfermagem (Simões, Alarcão, \& Costa, 2008).

Reforçamos o interesse formativo pelo ensino clínico porque, de acordo com Costa (2008), este é um contexto de aprendizagem privilegiado para a relação pedagógica e para a condução de aprendizagens que promovam a interligação da teoria à prática, com aprendizagem e exploração, enquanto método reflexivo sobre a realidade. Assim, como Serrano, Costa, e Costa (2011), que valorizam o contexto de ensino clínico, como um espaço importante de saberes e competências, promotor de uma prática reflexiva a partir da resolução de situações-problema. Desta forma, estas situações são momentos importantes que contribuem para a aquisição de conhecimento, permitindo aos estudantes desenvolverem um conjunto de saberes que proporcionem o cuidar em Enfermagem, a par da mobilização das aquisições em ensino teórico.

Nesta perspetiva, a Ordem dos Enfermeiros em 2002, pronunciando-se sobre os cuidados de qualidade, enunciava: "A qualidade exige, reflexão sobre a prática - para definir objetivos do serviço a prestar, delinear estratégias para os atingir - 0 que evidencia a necessidade de tempo apropriado para refletir nos cuidados prestados" (Ordem dos Enfermeiros, 2002, p.5). É neste sentido que a Ordem dos Enfermeiros propõe nos padrões de qualidade a explicitação e uniformização de ideias e critérios associados à caracterização do meta paradigma em Enfermagem, mobilizados na formação do $1^{\circ}$ ciclo. Torna-se importante refletir e valorizar com os estudantes o desenvolvimento das suas atividades em contextos da prática clínica, no confronto com os quadros de referência mobilizados no processo de formação, visando a reflexão da qualidade nos cuidados prestados. Por outro lado, os estudantes prestam cuidados através da caracterização do que designamos por processo de cuidados de acordo com Amendoeira (2000, p. 9) "como um processo de interação onde o centro de interesse é a pessoa e onde o profissional possui os conhecimentos específicos que lhe permitem fazer o diagnóstico e o planeamento do trabalho, que ele próprio executa e controla".

Torna-se assim indispensável explorar situações da prática de cuidados no processo de ensinoaprendizagem, de modo a permitir ao estudante o desenvolvimento do sentido crítico perante casos reais, levando a que passe da apreensão concreta de uma situação para a sua representação abstrata e conceptual (Carvalho, 2004; Abreu, 2007; Costa, 2008).

Nesta base de reflexão que temos vindo a descrever, realçamos as práticas no processo de cuidados e a respetiva conceptualizaçãa sobre as mesmas, procurando compreender que aquisições teóricas são desenvolvidas e como surgem enquadradas as conceções dos estudantes quando cuidam de pessoas. Tivemos presentes os princípios éticos e legais, suportados na autorização de acesso às fontes de dados numa escola de saúde, para consulta e análise documental, após obtenção do parecer favorável 
da comissão de ética para o desenvolvimento do estudo. Este foi desenvolvido tendo em consideração a necessária confidencialidade de dados e fontes que assegurámos ao longo de todo o processo de análise e tratamento dos dados.

No desenho do estudo optámos pelo estudo de caso, desenvolvendo uma estratégia metodológica, com recurso à análise de conteúdo e utilização do mapa conceptual, através do software CmapTools (Institute for Human and Machine Cognition. n.d.), para representar a conceção do processo de cuidados do estudante de Enfermagem em contexto clínico. Partimos de documentos de narração reflexões críticas elaboradas no mesmo contexto pelos estudantes do $4^{0}$ ano do $1^{\circ}$ ciclo do curso de Enfermagem.

Para a organização do material e para o processamento da leitura segundo critérios de análise, tivemos presente o que nos refere Bardin (2011) - as diferentes fases da análise de conteúdo organizam-se em torno de três polos desenvolvidos sequencialmente: a pré-análise; a exploração do material; o tratamento dos resultados, a inferência e a interpretação (Figura 1). A fase da pré-análise constituiu-se como a de organização, possuindo três missões: a escolha dos documentos a serem submetidos à análise; a formulação das hipóteses e dos objetivos; e a elaboração de indicadores que fundamentam a interpretação final. A escolha dos documentos (alínea a, na Figura 1) teve presente 0 critério da aleatoriedade (Beaud, 2003) para a seleção das reflexões críticas realizadas pelos estudantes do $4^{0}$ ano do curso de Enfermagem, num total de 30 documentos, resultantes de atividades de Enfermagem desenvolvidas em contexto de ensino clínico.

Definida a constituição do corpus da análise (alínea b, Figura 1), estabelecemos contacto com os documentos a analisar e realizámos a leitura flutuante, no sentido de retirar impressões e orientações em função das hipóteses emergentes (Bardin, 2011). Neste momento foi fundamental a leitura dos documentos, interrogarmo-nos sobre o que era sugerido a priori face ao processo de cuidados desenvolvido pelo estudante e pelo conhecimento que possuíamos, tendo subjacente os conceitos organizadores (alínea d, Figura 1): formação em Enfermagem; estudante; ensino clínico; pessoa; e cuidados de Enfermagem. Do quadro teórico que mobilizámos constituíram- se em indicadores de análise as seguintes palavraschave (alínea e, Figura 1): aprendizagem; cuidados; procedimentos; estratégias; avaliação; reflexão; responsabilidade; (...). A preparação do material (alínea c, Figura 1) consistiu na numeração de todos os documentos, dispondo de colunas vazias à direita e à esquerda para notas e códigos. Quanto às regras de categorização e codificação (alínea f, Figura 1), tivemos presente na codificação a escolha do recorte da unidade de registo, sendo esta considerada a unidade de significação de nível semântico, a palavra. Há pois que ter em conta que todas as palavras do texto podem ser levadas em consideração ou podemos reter unicamente as palavras-chave ou as palavras-tema, ou ainda efetuarmos a análise de uma categoria de palavras (Bardin, 2011). Nesta perspetiva valorizámos, num primeiro momento, todas as palavras com sentido semântico idêntico que pudessem constituirse em dimensões importantes quando pensamos na conceção do processo de cuidados desenvolvido pelos estudantes. Quanto à unidade de contexto, considerámos ser a frase para a palavra como unidade de compreensão para codificar a unidade de registo, cujas dimensões superiores às unidades de registo são ótimas para compreendermos a significação exata da palavra, podendo ser sinónimas ou próximas a nível semântico. As técnicas desenvolvidas (alínea g, Figura 1) foram testadas de forma manual e informática no sentido de assegurar a sua correta aplicação, segundo as possibilidades de leitura do computador e dos programas utilizados.

As diferentes operações da pré-análise (Figura 1) foram sendo desenvolvidas, ficando a fase de análise propriamente dita na aplicação sistemática das decisões tomadas. Esta fase longa consistiu essencialmente em operações de codificação e decomposição em função das regras previamente formuladas (Bardin, 2011, p. 127): a exploração do material fizemo-la para identificar as dimensões que os estudantes valorizam na conceção do processo de cuidados desenvolvido em ensino clínico. Procedemos à identificação de palavras e/ou expressões significativas, importantes para a definição das unidades de análise. Deste procedimento, surgiram um conjunto de palavras com significado, enquadrado ao conceito de processo de cuidados.

Realizámos a agregação das palavras, por aproximações semânticas ligeiras (cuidar, cuidado, cuidados ou prática, práticas), mas não destituídas 
de critérios de agrupamento (o cuidar, a prática de cuidados), permitindo, deste modo, classificar a informação através de uma lista de palavras com sentido semântico, num total de 34 que se denominaram palauras plenas, isto é, palavras portadoras de sentido (Bardin, 2011). Valorizámos as unidades de enumeração porque pretendíamos a frequência com que as expressões se repetiam, no sentido de ajudar a perceber a intensidade das palavras e o sentido que os estudantes lhes conferiam. Com o recurso ao software do Microsoft Word 2010, submetemos a lista de todas as palavras plenas ao corpus de análise. O procedimento consistiu em submeter palava a palavra ao documento em formato digital, através da opção localizar seguida da localização avançada, obtendo-se a identificação da palavra e o número de vezes que a mesma se repete.
Com a localização da palavra, na unidade de contexto, efetuámos a descontextualização através da regra do recorte. O recorte da unidade de registo foi efetuado de acordo com os referenciais teóricos, com o sentido atribuído, ficando ordenadas as várias unidades de contexto numa tabela de dupla entrada em suporte digital. Este procedimento foi replicado para as 34 palavras submetidas. Posteriormente realizámos nova decomposição, através do recorte e aglutinação de cada palavra plena numa tabela única. Reconstruímos assim, uma tabela individual para cada palavra plena. Obtivemos deste modo a (re)contextualização através da análise das diferentes unidades de contexto que integram a totalidade das palavras plenas, de sentido semântico igual. Para além da frequência obtida fomos ainda sensíveis ao seu sentido semântico, procurando a sua respetiva contextualização teórica.

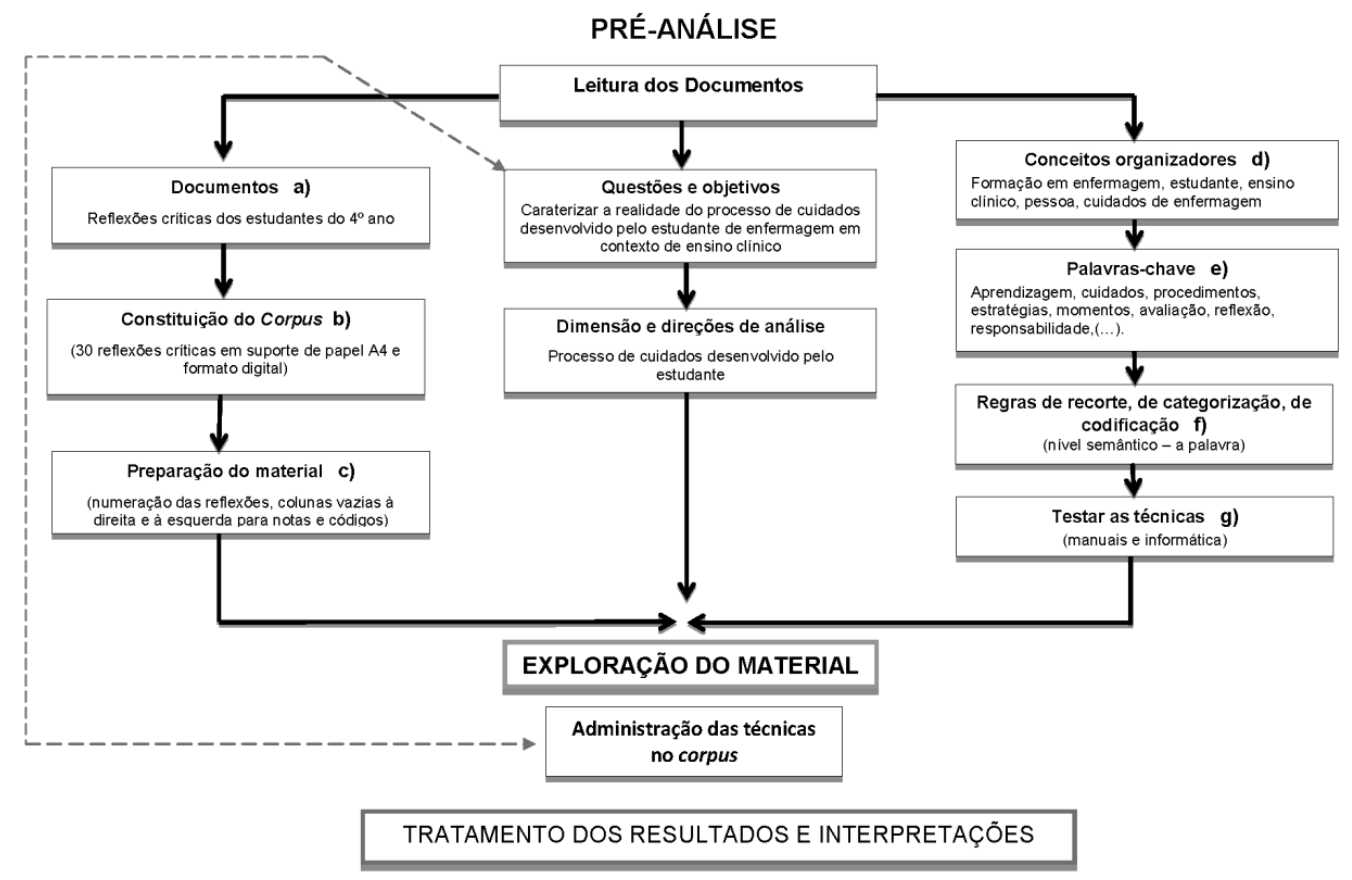

Esquema adaptado de Bardin, 2011

Figura 1. Desenvolvimento das fases da análise do estudo

Neste sentido, dando continuidade à representação da estrutura cognitiva do processo de cuidados desenvolvido pelos estudantes, através das unidades semânticas já constituídas pelas palavras plenas identificadas, recorremos ao mapa conceptual como instrumento epistemológico, porque, de acordo com Carvalho (2009), ajuda na compreensão da relação entre o domínio conceptual e o domínio factual do processo científico que pretendemos.

Por outro lado, reconhecemos o mapa conceptual como uma ferramenta cientifico-pedagógica, relevante no ensino de Enfermagem, capaz de promover aprendizagens significativas (Fonseca, Extremina, Ferreira, \& Leite, 2010). Todos estes 
aspetos se suportam nas evidências encontradas (Clayton, 2006) quando identificamos a utilização dos mapas conceptuais para avaliação de conhecimentos e habilidades de pensamento crítico dos estudantes bem como na avaliação do desempenho clínico.

Uma vez que nos focalizámos neste estudo na especificidade do processo de cuidados desenvolvido pelos estudantes, pretendemos com a mobilização do mapa conceptual representar o seu conhecimento. Fazemo-lo na lógica da associação da teoria à prática, para clarificação de conceitos-chave, estabelecendo relações significativas entre palavras já identificadas e o sentido que os estudantes lhe atribuem através de proposições.

Trata-se, portanto, de um procedimento que num primeiro momento se constituiu em agrupar todas as palavras plenas através do referenciamento constante nos encadeamentos: palavra plena - palavra instrumento (palavra de ligação) permitindo formar várias relações com significado, que possibilitaram a construção de uma rede semântica e composição do mapa conceptual, com recurso ao software Cmaptools.

$\mathrm{Na}$ perspetiva compreensiva dos dados, procedemos à analise da rede semântica através dos conceitos, das palavras, frases e/ou proposições significativas, no sentido da organização do conhecimento dos estudantes face à conceção do processo de cuidados. A análise desta rede permite assim representar as palavras plenas portadoras de maior sentido, como os conceitos mais gerais: cuidados; pessoa; intervenção; momentos; ajudar; aprendizagem; reflexão; competências; e avaliação. Tal aspeto ocorre no sentido da compreensão do conhecimento dos estudantes, integrando as restantes palavras plenas, como os conceitos menos gerais na representação hierárquica do mapa conceptual.

Visando a estrutura do tratamento dos resultados e interpretações (Figura1), foi fundamental analisar os dados dos quadros de codificação obtidos do conteúdo do mapa conceptual, tendo subjacente o referencial teórico e o objetivo do estudo. Desta forma foi possível encontrar a linha semântica que os conduzia, relacioná-los uns com os outros, integrando as qualidades da análise de conteúdo e do mapa conceptual. Tornou-se deste modo possível a identificação e representação dos conceitos, com significado atribuído à conceção do processo de cuidados desenvolvido em ensino clínico pelo estudante de Enfermagem.

Neste sentido, o processo de associação de palavras com recurso ao mapa conceptual, para determinação e indicação de correspondências entre as mensagens dos estudantes e a realidade subjacente, foi fundamental para avaliação das unidades da rede semântica (Jonassen, 2007). Valorizámos aqui a centralidade de cada nó desta rede, pelo número de ligações diretas (os nove conceitos mais gerais) e de ligações indiretas (conceitos menos gerais e outros a eles diretamente ligados). Considerámos desta forma a centralidade como um critério para a identificação da importância destes conceitos para o processo de cuidados pelo estudante, estruturando-a a partir dos conceitos centrais e das suas relações. Com as setas colocadas na Figura 2, pretendemos tornar visíveis as relações hierárquicas e/ou causais encontradas. De acordo com Jonassen (2007), a quantidade e a precisão dos pares de palavras ligadas e a quantidade de ligações entre os pares de palavras indicam-nos uma compreensão profunda das mesmas. Valorizámos estes aspetos para a compreensão do conhecimento dos estudantes e para a identificação da sua conceção no âmbito do processo de cuidados.

Quando definimos a palavra pessoa valorizámos uma ligação causal, que integra a individualidade (conforme Figura 2), estendendo-se para nova ligação: os conbecimentos; disponibilidade; comunicação; e para a palavra escuta. Do mesmo modo, na palavra plena cuidados verificámos uma relação causal, mas representada de forma hierárquica, sendo uma palavra que apresenta dois nós de correspondências, um que integra a continuidade, planeamento e a individualidade, e outro dirigido para a palavra prestação, que dirige para as prioridades, competências e qualidade. Na palavra intervenção, verificámos uma relação causal, que permitiu a representação de acordo com os estudantes com qualidade; verificámos dois nós de correspondências, um com a palavra responsabilidade e outro com prática, através de procedimentos e tarefas. Para a palavra aprendizagem, identificámos uma relação causal que ocorre através de experiências vividas e de oportunidades, permitindo a reflexão e a autoavaliação. 


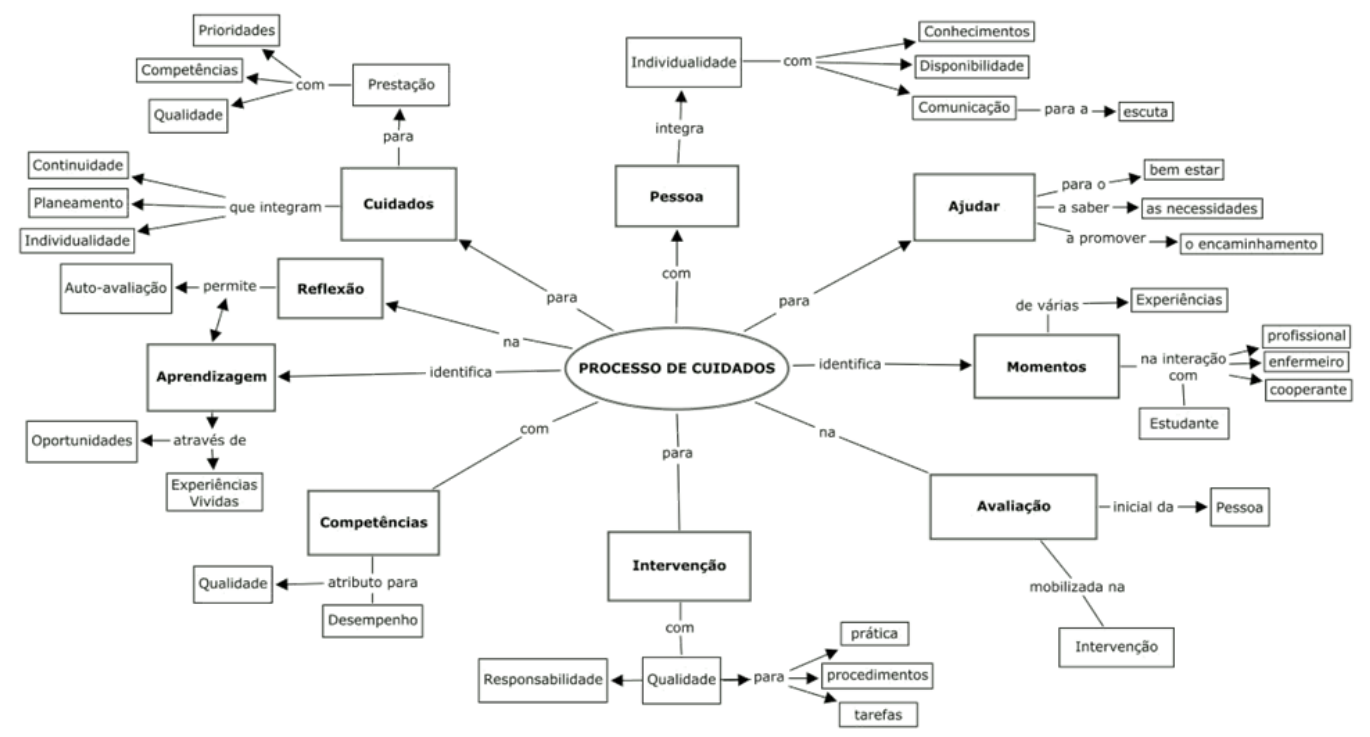

Figura 2. Mapa conceptual que representa os conceitos do processo de cuidados desenvolvido pelo utente

Neste sentido, através da identificação das relações entre os conceitos mais gerais, da natureza dessas relações com os outros diferentes conceitos, foi possível representarmos através do mapa conceptual o que os estudantes integram como dimensões da conceção do processo de cuidados desenvolvido em contexto de ensino clínico. Foi ainda possível de modo lógico com todos os conceitos (Carvalho, 2009; Jonassen, 2007) organizá-los de forma a enquadrar a temática da conceção do processo de cuidados desenvolvido pelo estudante, em três temas representativos: conceção dos cuidados; intervenção de Enfermagem; e aprendizagem do processo de cuidados. Situámo-nos assim numalinha de pensamento que valoriza o método da disciplina de Enfermagem através do processo de planeamento das atividades de Enfermagem, com intervenção no sujeito de cuidados e com a consequente avaliação. Sendo que a aprendizagem pressupõe igualmente avaliação em contexto de ensino clínico, considerámo-la num duplo sentido ao olharmos para o estudante: por um lado o processo de ensino-aprendizagem per si e por outro o processo de cuidados por ele desenvolvido em interação com a pessoa alvo dos cuidados, que é inerente à primeira dimensão.

Desta forma, assumimos os resultados obtidos como sobreponíveis aos temas teoricamente enquadrados para o Processo de Cuidados, parecendo evidenciar-se alguma coerência entre as aquisições em ensino teórico e as conceções assumidas pelos estudantes no ensino clinico.

\section{Conclusão}

Neste estudo tivemos presente uma abordagem compreensiva da identificação das dimensões que os estudantes de Enfermagem integram na conceção do processo de cuidados em ensino clínico.

As dimensões identificadas pelos próprios estudantes no âmbito do conceito processo de cuidados foram: cuidados; pessoa; ajudar; momentos; avaliação; intervenção; competências; aprendizagem; e reflexão. Tendo estas dimensões presentes e porque pretendíamos a perspetiva cognitiva dos estudantes, a representação assumida afigurou-se em três temas: conceção dos cuidados; intervenção de Enfermagem; e aprendizagem do processo de cuidados. Nesta perspetiva, os estudantes enfatizam a organização do planeamento dos cuidados de Enfermagem na procura do momento adequado para a intervenção à pessoa alvo de cuidados. Por outro lado, valorizam a aprendizagem do processo de cuidados com enfoque à reflexão e responsabilidade sobre as práticas de cuidados. Estes aspetos, considerados como conceitos integradores no ensino teórico, salientam também para o contexto de ensino clínico uma apropriação de conhecimentos alicerçada na integração e transposição de saberes visível na prática clínica dos estudantes.

Neste contexto, reconhecemos a estratégia metodológica do mapa conceptual utilizada neste estudo como um importante instrumento de 
desenvolvimento analítico, no sentido em que nos revelou potencial para representar o domínio do conhecimento dos estudantes face ao processo de cuidados, identificando as suas diferentes dimensões. Pretendemos rentabilizar os resultados deste estudo na aferição das conceções do processo de cuidados (pelos estudantes) para uma prática clínica mais refletida, com espaço à mobilização de evidências e apelo ao pensamento crítico para um adequado julgamento clínico em Enfermagem. Estes aspetos surgem como possíveis através da adequação de estratégias pedagógicas nos vários contextos de formação, com momentos em seminário, promotores da reflexão sobre as práticas, com recurso às narrativas, através da pesquisa e análise informática como meio para o desenvolvimento de novos conhecimentos e do saber contextualizado dos estudantes.

As implicações emergentes para a formação no $1^{\circ}$ ciclo de Enfermagem estruturam-se em dois níveis de interesse: ao nível metodológico, sendo que valorizámos a necessidade de identificar a natureza das relações semânticas entre as dimensões/ conceitos de forma mais intensiva, na procura da organização do conhecimento dos estudantes, mobilizando a informação obtida num quadro conceptual progressivamente mais complexo; e ao nível do processo pedagógico, em que valorizámos a utilização do mapa conceptual como estratégia para a promoção da aprendizagem dos estudantes. Emerge também como relevante na promoção da autorreflexão e na aquisição de competências ao nível do pensamento crítico nos estudantes.

\section{Referências Bibliográficas}

Abreu, W. (2007). Formação e aprendizagem em contexto clínico: Fundamentos, teorias e considerações didácticas. Coimbra, Portugal: Formasau.

Amendoeira, J. (2000). Cuidado de Enfermagem. Intenção ou acção. O que pensam os estudantes? Nursing, 5, 8-14.

Amendoeira, J. (2004). Enfermagem em Portugal. Contextos, atores e saberes. Enfermagem, 2(35/36), 13-22.

Amendoeira, J. (2009). Ensino de Enfermagem: Perspectivas de desenvolvimento. Pensar Enfermagem, 13(1), 2-12.

Bardin, L. (2011). Análise de conteúdo (5 ed). (L. Pinheiro, Trad.). Lisboa, Portugal: Edições 70.

Beaud, J. P. (2003). A amostragem. In B. Gauthier (Ed.), Investigação social: Da problemática à colheita de dados (3nd ed., pp. 201-232). Loures, Portugal: Lusociência.
Carvalho, A. L. (2004). Avaliação da aprendizagem em ensino clinico no curso da licenciatura em Enfermagem. Lisboa, Portugal: Instituto Piaget.

Carvalho, J. E. (2009). Metodologia do trabalho científico: «Saber - fazer» da investigação para dissertações e teses (2a ed.). Lisboa, Portugal: Escolar Editora.

Clayton, L. H. (2006). Concept mapping: An effective, active teaching-learning method. Nursing Education Perspectives, 27(4), 197-203.

Costa, A. (2008). Aprender a cuidar: Consonâncias e dissonâncias de um binómio desafiante. In L. M. Gomes (Coord.), Enfermagem e úlceras de pressão: Da reflexão sobre a disciplina às evidências nos cuidados (pp. 81-102). Islas Canarias, Espanha: ICE.

Decreto-lei no 115/13 de 7 de Agosto (2013). Diário da Republica $n^{\circ}$ 151/13, I Série - A. Ministério da Educação e Ciência. Lisboa, Portugal.

Fonseca, A. P., Extremina, C. I., Ferreira, A., \& Leite, C. (2010). Aplicações dos mapas conceptuais no ensino Universitário Pós-Bolonha: Os mapas como ferramentas para o aumento de eficiência do processo de ensino aprendizagem. Recuperado de http://repositorio-aberto.up.pt/bitstream/ 10216/35056/2/69655.pdf

Institute for Human and Machine Cognition. (n.d.). Cmap Tools: Software para a construção de mapas conceptuais. Recuperado de http://cmap.ihmc.us

Jonassen, D. H. (2007). Computadores, ferramentas cognitivas: Desenvolver o pensamento crítico nas escolas ( $2^{\mathrm{a}} \mathrm{ed}$.). (A. Gonçalves, S. Fradão, \& M. Soares, Trad.). Porto, Portugal: Porto Editora.

Josso, M. C. (2002). Experiências de vida e formação. Lisboa, Portugal: Educa.

Ordem dos Enfermeiros. (2002). Padrões de qualidade dos cuidados de Enfermagem. Enquadramento conceptual. Divulgar. Lisboa, Portugal: Autor.

Ordem dos Enfermeiros. (2007). Enfermagem portuguesa: Implicações na adequação ao processo de Bolonha no atual quadro regulamentar. Lisboa, Portugal: Autor.

Rua, M. (2011). De aluno a enfermeiro: Desenvolvimento de competências em contexto de ensino clinico. Loures, Portugal: Lusociência.

Serrano, M. T. P., Costa, A. S. M. C., \& Costa, N. M. V. N. (2011). Cuidar em Enfermagem: Como desenvolver a(s) competência(s). Revista de Enfermagem Referência, 3(3), 15-23. doi:10.12707/RIII1019

Simões, J. F., Alarcão, I., \& Costa, N. M. V. N. (2008). Supervisão em ensino clínico de Enfermagem: A perspetiva dos enfermeiros cooperantes. Revista de Enfermagem Referência, 2(6), 91108.

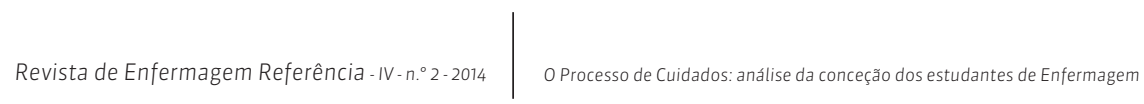

